\title{
Gynecologic ultrasonography: recent advances and research in various technical modalities
}

This article was published in the following Dove Press journal:

Reports in Medical Imaging

14 October 2010

Number of times this article has been viewed

\section{Juraj Drobný}

First Department of Obstetrics and Gynaecology, St. Cyril and Method University Hospital, Bratislava, Slovak Republic
Correspondence: Juraj Drobný Mlynarovicova 24, 85I03 Bratislava, Slovak Republic

Tel +42 1903566837

Fax +42 I26 23। 5I 40

Email drobny715@gmail.com

\begin{abstract}
This paper reviews clinical research in gynecologic sonography, focussing on uterine cavity lesions, endometrial abnormalities and adnexal masses (including endometriosis), and ectopic pregnancy. For each topic, detection of sonographic pathologic features and sonographic mode are discussed, as well as the latest applications of sonodiagnostic methods, and relevant topics in clinical research. A new approach to evaluation of sonographic structures can be seen, including for borderline mucinous and serous ovarian tumors, in mean gray value, evaluation of grade of tissue echogenicity, evaluation of intact endometrial midline echo in ectopic pregnancy, and application of gel instillation sonography. Novel sonographic three-dimensional modalities, such as virtual navigation through three orthogonal planes, multislice tomosonography, volumetry by a virtual organ computer-aided analysis system, three-dimensional power Doppler, and space reconstruction of structures enable gynecologic diagnoses to be made more exactly. Clinical research investigates different sonographic features in benign and malignant gynecologic pathology. For studies of typical signs of benign uterine fibroids, endometrial volume, and vascularization of malignant endometrial tumors, as well as typical benign adnexal structures, the ovarian crescent sign were performed. At this time, no exact sonographic features for distinguishing between benign and malignant gynecologic tumors are available.
\end{abstract}

Keywords: sonography, uterine cavity lesions, endometrial abnormalities, adnexal masses

\section{Introduction}

Sonography has become the most important imaging method in gynecologic pathology. The main objectives of this examination are to assess the likelihood of uterine and ovarian malignancy and to search for the cause of infertility. These problems are connected with the presence of uterine/endometrial abnormalities and adnexal masses.

An appropriate sonographic mode and evaluation of sonographic features are the most frequently discussed problems in connection with sonographic gynecologic diagnosis. The quality of detection of each sonographic entity depends on the sonographic modality used which gives the most relevant information. Exact explanation of sonographic findings is also crucial for performing invasive procedures, surgery, follow-up, and prognosis.

New sonographic modalities, recent research, and relevant visions are presented yearly by opinion leaders at the world congress of the International Society on Ultrasound in Obstetrics and Gynecology (ISUOG). In this paper, important sonographic information on uterine cavity lesions, endometrial abnormalities, and adnexal masses, including endometriosis and ectopic pregnancy, are presented, as well as the 
conclusions of the 2009 World Congress of the ISUOG in Hamburg. Recent clinical research is also discussed.

\section{Uterine cavity lesions}

Myoma, polyps, and Müllerian anomalies, ie, septate uterus, are the most frequently detected sonographic pathologic features in the uterine cavity causing infertility (Figure 1). The sonographic features of uterine myoma can usually be evaluated reliably. More echogenic ovoid structures are distinct from less echogenic myometrial tissue of the uterine wall. Dilatation of the uterine cavity by fluid instillation enables detection of both entities, uterine septum, arising from the fundal area, dividing cornua, and an endometrial polyp by depiction of the feeding vessels.

There is a lack of consensus between sonologists on the definition of an intracavity lesion, as well as the definition of a smooth versus irregular outline. ${ }^{1}$ The International Endometrial Tumor Analysis group, created at ISUOG 2008, has proposed terms and definitions to describe ultrasound findings in the uterine cavity. The following terms should be used for uterine cavity lesions: the echo formed by the interface between an intracavitary lesion and the endometrium is the "bright edge"; a fluid-filled uterine cavity endometrial polyp is defined as "extended" if its basis involves more than $25 \%$ of the endometrial surface and "localized" if it involves less than $25 \%$. Moreover, a polyp is described as "pedunculated" if the ratio between the diameter of the base and the maximal transverse diameter is $<1$ and "sessile" if it is $\geq 1$. The proportion of submucous fibroid that projects into the uterine cavity, ie, "grading", should be depicted as Grade 0 (G0) when the fibroid completely fills the uterine cavity, $\mathrm{G} 1$ if the endocavitary part is $>50 \%$, and G2 if $<50 \%$. The echogenicity of both lesions is defined as "uniform" or "nonuniform" and the outline as "regular" or "irregular". 2

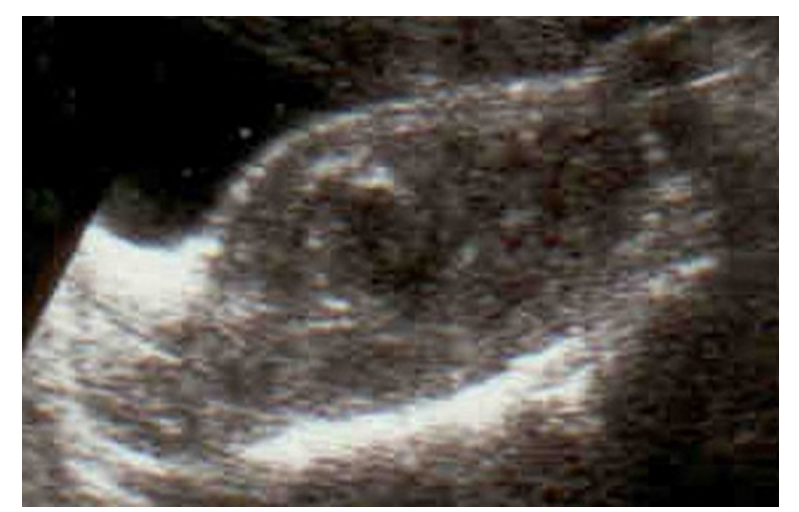

Figure I Uterine cavity lesion (fibroid).
Two-dimensional and three-dimensional transabdominal sonography and transvaginal sonography, two-dimensional saline infusion sonography, or hystero-contrast-sonography (HyCoSy), are commonly used to detect these lesions. The three-dimensional method seems to be a useful complement to the two-dimensional method, ${ }^{3}$ and tubal patency can be examined simultaneously.

\section{Current examinations}

\section{Gel instillation sonography}

Gel instillation sonography, as well as three-dimensional color Doppler and power Doppler, have enhanced the ability to diagnose uterine cavity lesions. Although there are some advantages of saline infusion sonography, gel instillation sonography seems to be a better method for this diagnosis. Gel instillation sonography has increased the level of diagnostic confidence with regard to the presence of an intracavity lesion. ${ }^{4}$ The diagnostic accuracy of gel instillation sonography is comparable with that of saline infusion sonography. ${ }^{5} \mathrm{Gel}$ instillation sonography is an alternative to saline infusion sonography. ${ }^{6}$ The image quality of saline infusion sonography is slightly better than gel instillation sonography. ${ }^{7}$ Three-dimensional multislice saline infusion sonography can accurately diagnose and classify submucous myoma. ${ }^{8}$ Three-dimensional saline infusion sonography is an appropriate method to identify submucous fibroids for transcervical resection. ${ }^{9}$ Unlike saline infusion sonography, gel instillation sonography does not affect detection of the vessels feeding endometrial polyps..$^{10}$

\section{Three-dimensional sonography}

Three-dimensional transvaginal sonography allows better space orientation than the two-dimensional technique, which is especially important in this kind of uterine deformity. Three-dimensional transvaginal sonography in the coronal view allows detection of different types of uterine septa. ${ }^{11}$ The compliance of transvaginal sonography is higher for endometrial polyps than for submucous fibroids. Diagnosis of endometrial polyps by transvaginal sonography is better in premenopausal women. ${ }^{12}$ In infertile women with suspected uterine cavity lesions, three-dimensional transvaginal sonography in the coronal plane is as sensitive as three-dimensional saline infusion sonography, and both are as accurate as diagnostic hysteroscopy. ${ }^{13}$

\section{Magnetic resonance imaging}

In certain situations, other imaging procedures are more suitable. Magnetic resonance imaging examination improves 
the quality of pretherapeutic mapping for submucosal fibroids. ${ }^{14}$

\section{Tubal patency}

Flow through the fallopian tube after fluid instillation in the uterine cavity can be used to detect tubal patency, and is commonly used to diagnose infertility. HyCoSy particles and saline solution are the fluids commonly used for this purpose. Recently, new sophisticated methods to visualize tubal patency have been used. Automated three-dimensional contrast-enhanced ultrasound technology coded contrast imaging by HyCoSy allows for reliable assessment of tubal patency. ${ }^{15}$

The ISUOG congress in 2009 showed that introduction of new imaging methods will change our approach to detection of uterine cavity lesions. The first step is basic orientation and diagnosis by two-dimensional sonography and the next is fluid instillation in the uterine cavity. Dilatation of the uterine cavity allows us to see the inside structures better. Just by pushing another button without any other procedure, it is possible to see the three-dimensional space orientation with more precise shape and location of structures. On the other hand, fluid instillation procedures are more sophisticated. Replacement of fluid by gel seems to be more suitable for performing the procedure and evaluating findings more precisely. Computerization of flow with echocontrast particles enhances the diagnosis, particularly of tubal patency.

\section{Clinical research}

The aim of current research is to enable distinction between malignant and benign lesions. The first step is to establish if a tumor is benign. Thus, the main topic of interest in the area of uterine cavity lesions is the distinction between an endometrial polyp and submucosal fibroid, and also with the successive surgical procedures. In some cases, sonographic pictures of both entities can be confused. A polyp with a broad base can look like a myoma, and an intracavitary fibroid with a tiny stalk may appear as an endometrial polyp.

Doppler flowmetry seems to resolve this problem. Previously, Alcazar et al depicted a single vessel penetrating from the endometrium to an endometrial polyp using color Doppler. ${ }^{16}$ This characteristic sign, ie, a single-vessel pattern, was confirmed by Timmerman et al as the "pedicle artery" sign. ${ }^{17}$ On the other hand, the color Doppler characteristics of fibroids are not so specific. The multiple-vessel and scatteredvessel patterns shown by Alcazar et al can occur not only with a benign fibroid, but also with a malignant lesion, eg, endometrial cancer, in this location. ${ }^{16}$
Current research is focused on the application of power Doppler sonographic examination to get a specific picture of fibroids. This approach allows us to use color Doppler to depict low-velocity flow. Processing of the amplitude of Doppler signal, rather than the Doppler frequency shift, like in color Doppler, enables power Doppler to visualize small vessels. Cil et al have identified a so-called "rim-like" vessel pattern, characteristic of fibroids. Unlike the single central, circular, or semicircular vessel surrounding the focal endometrial lesion, a few small, scattered accompanying vessels may sometimes create this pattern. ${ }^{18}$

\section{Endometrial abnormalities}

Thickness of the endometrium and myometrial invasion is important information for detecting endometrial malignancy (Figure 2). An International Endometrial Tumor Analysis

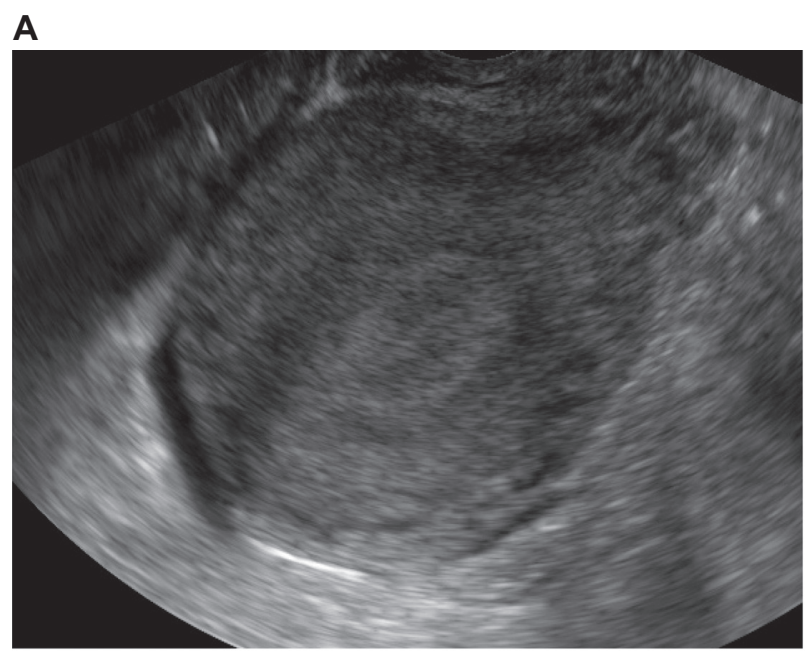

B

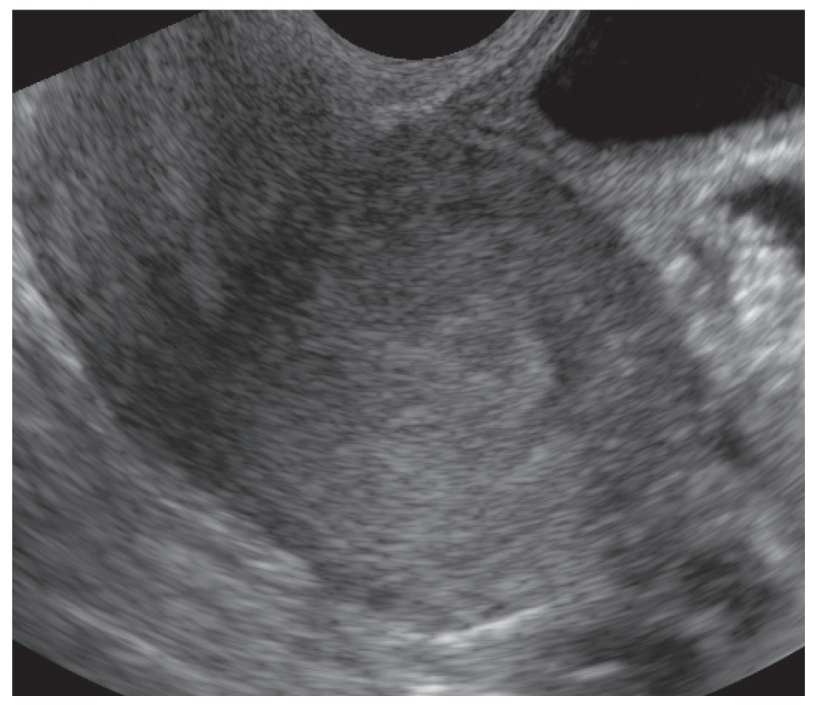

Figure 2 Endometrial abnormalities. A) Normal uterus with normal three-layered endometrium. B) Myometrial invasion. 
group statement has now proposed terms, definitions, and measurement techniques for endometrial abnormalities. Endometrial thickness assessment consists of measuring endometrial layers in the sagittal plane, perpendicular to the endometrial midline, and calipers are placed at the level of the two opposite most distant endometrial-myometrial interfaces. When intracavitary fluid is present, the only appropriate result is the sum of both single layers, without fluid packet measurement. Qualitative assessment of the endometrium involves several sonographic parameters, ie, the echogenicity of the endometrium can be "hyperechogenic", "isoechogenic", or "hypoechogenic" compared with the echogenicity of the myometrium. The sonographic structure of the endometrium is defined as "uniform" if the three-layered endometrium appears homogeneous, and as "nonuniform" if it appears heterogeneous. The endometrial midline is "linear" or "nonlinear" if an interface is seen and "irregular" or "not defined" in the absence of a distinct interface. The endometrial-myometrial junction can be "regular", "irregular", "interrupted", or "not defined". 2

Two-dimensional sonographic biometry is usually used as a relatively good approach to measure thickness of the endometrium. On the other hand, this sonographic parameter is not sufficient for evaluation of malignant disease of the endometrium. In the case of malignant infiltration of the endometrium into the myometrium, exact sonographic detection of the border between these two tissues is of great interest. However, current equipment does not offer sufficient information, and even the three-dimensional is not superior to the two-dimensional mode in endometrial malignancy detection. ${ }^{19}$

Recently, improvements in current methods have been notable. In addition, many three-dimensional modes, with color and power Doppler, enable research on the growth of malignant endometrial masses and their penetration into the myometrial layer.

\section{Improvements in current detection methods}

Endometrial thickness measurement with fluid contrast ultrasound (saline infusion sonography) can be used to detect endometrial abnormalities. ${ }^{20}$ Saline infusion sonography with liquid-based endometrial cytology is an accurate diagnostic tool for postmenopausal uterine bleeding. ${ }^{21}$

Endometrial volume and three-dimensional power Doppler analysis are good diagnostic tools for predicting endometrial carcinoma and hyperplasia. ${ }^{22}$ Uterine virtual navigation through three orthogonal planes using three-dimensional ultrasound is a reliable method for assessing myometrial infiltration in patients with endometrial cancer. ${ }^{23}$ Virtual organ computer-aided analysis (VOCAL) is an appropriate method for evaluation of vascularization of the endometrium and of the endomyometrial junction. ${ }^{24}$ In comparison with other imaging methods, three-dimensional ultrasound is more sensitive than magnetic resonance imaging for the prediction of myometrial invasion of endometrial cancer. ${ }^{25}$

The ISUOG congress in 2009 focused on insufficient information on endometrial thickness from the malignancy point of view. There were opinions presented that fluid instillation enables enhancement of sonographic knowledge about the endometrium, especially by additional cytology examination. On the other hand, volumetric measurements and power Doppler examination in the three-dimensional mode have the potential to contribute to diagnosis of endometrial cancer.

\section{Clinical research}

Discrimination between benign and malignant endometrium by sonography is of great interest for diagnosis of noninvasive endometrial cancer. Growth of endometrial tissue mass and tumor neovascularization are the most appropriate parameters for this investigation. Endometrial thickness measurement and Doppler flowmetry by two-dimensional sonography show thicker endometrium with enhanced flow in malignant transformation of endometrium. ${ }^{26}$ On the other hand, no objective and reproducible parameters to distinguish between these entities has been established.

Current research is using newer sonographic threedimensional methods which enable exact measurement not only of the volume of chosen structures by the VOCAL system but also of the "density" of neovascularization by power Doppler angiography. A multiplanar system with outlining of the endometrial-myometrial junction measures the volume of endometrium, and three vascularity indices, ie, vascularization index, flow index, and vascularization flow index, are calculated automatically.

Opolskiene et al showed that endometrial volume was larger and that flow indices are higher in patients with a malignant endometrium than in those with a benign endometrium, but there is substantial overlap between the two categories. Three-dimensional ultrasound imaging was not superior to that of endometrial thickness as measured by twodimensional ultrasound examination, and three-dimensional power Doppler imaging added little to information on endometrial thickness and volume. ${ }^{19}$

Galván et al demonstrated that there were no statistically significant differences regarding endometrial thickness 
according to tumor type and grade. However, tumors with deep myometrial infiltration and at an advanced stage had a significantly thicker endometrium. There was no statistical difference in endometrial volume according to tumor histologic type. However, endometrial volume was significantly higher in poorly differentiated tumors and deeply invasive tumors. Regarding three-dimensional power Doppler analysis, as with endometrial volume, there were no statistical differences for vascularization index, flow index, and vascularization flow index according to tumor histologic type, and all three parameters had significantly higher values in poorly differentiated tumors and deeply invasive tumors. In conclusion, three-dimensional power Doppler analysis of tumor vascularization in endometrial cancer correlates with some prognostic histologic characteristics. ${ }^{27}$

\section{Adnexal masses}

Cystic, solid and complex structures localized in the pelvis laterally from uterus are the most common features in both benign and malignant adnexal masses (Figure 3). Due to both the irregularity of adnexal structures and their close contact with the normally irregular intestinal structures, exact detection of adnexal masses is sometimes confusing. On the contrary, peristaltic movements distinguish both structures. Tumor neovascularization can be detected by Doppler examination. Because of lack of specificity, the current approach does establish malignancy. Scoring systems using mathematic models for structural changes are not successful either. Serum oncomarker level determination, especially of CA 125, in patients with ovarian tumors enables an improved detection rate for ovarian malignancy ${ }^{28}$ In ultrasound characteristics of malignant and benign adnexal masses, assessment of blood flow at color Doppler ultrasonography and wall irregularity have shown the highest intercenter variability. ${ }^{29}$ Volume evaluation of pathologic and normal ovaries by twodimensional three distances and three-dimensional VOCAL software are similar. ${ }^{30}$ The logistic regression model cannot help to discriminate between benign and malignant unilocular solid adnexal masses. ${ }^{31}$

Endometrioma (Figure 4a), a form of ovarian endometriosis, is a specific type of adnexal morphology. A unilocular cyst with ground-glass echogenicity of cyst fluid is a characteristic sonographic picture of this lesion..$^{32}$ Transvaginal sonography is the most suitable approach. The adenomyotic retroperitoneal form of endometriosis is characterized by different sonographic parameters from that of endometrioma (Figure 4b). This lesion is localized in the uterine wall, in the septum vesicovaginale or septum rectovaginale, its shape
A

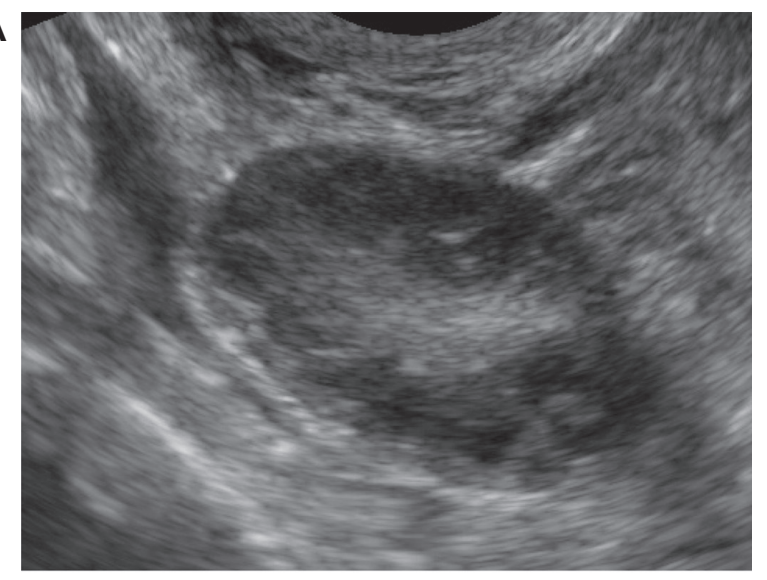

B

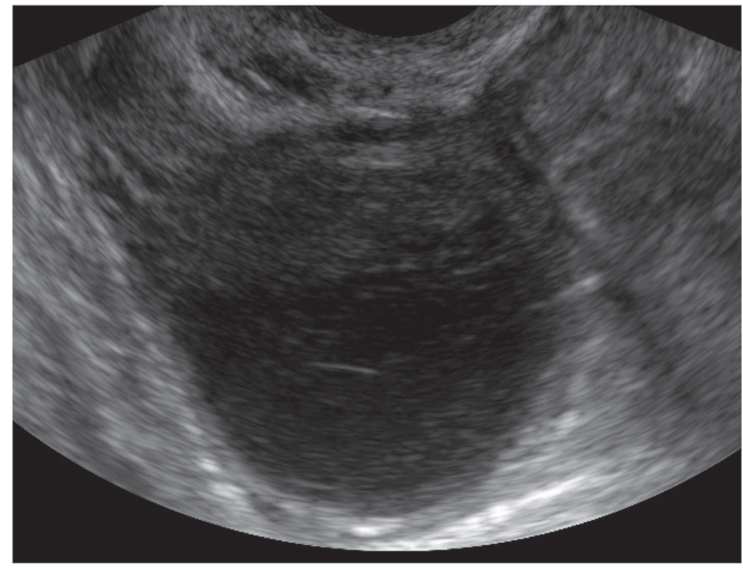

C

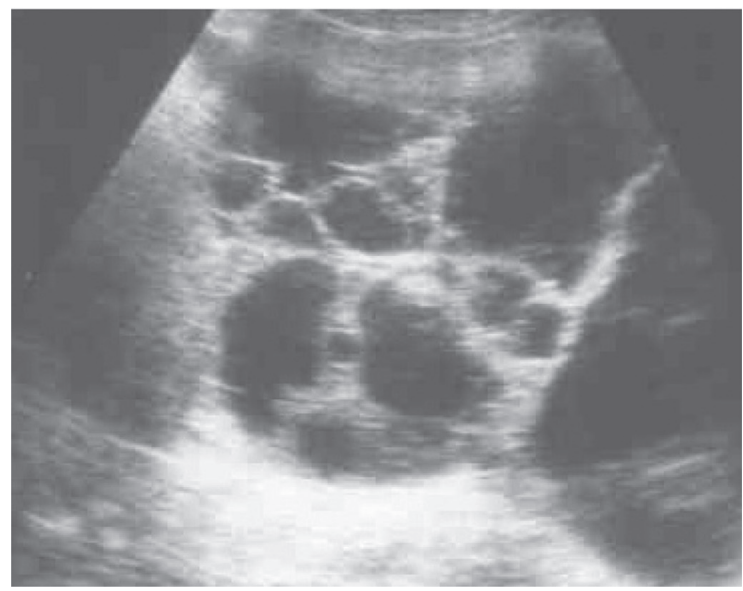

Figure 3 Adnexal masses. A) Normal ovary. B) Simple ovarian cyst. C) Multilocular ovarian cyst.

is ovoid and less regular, and the content is more echogenic, with a diameter usually of $1-5 \mathrm{~cm}$. Exact diagnosis is important, especially for minimally invasive surgery. ${ }^{33}$ Transvaginal sonography and transrectal sonovaginography with saline instillation into the vagina can be used. Peritoneal endometriosis is not detectable by current ultrasound equipment. Ovarian endometriosis, similar to peritoneal or retroperitoneal endometriosis, is the reason for both chronic pelvic pain and infertility. 


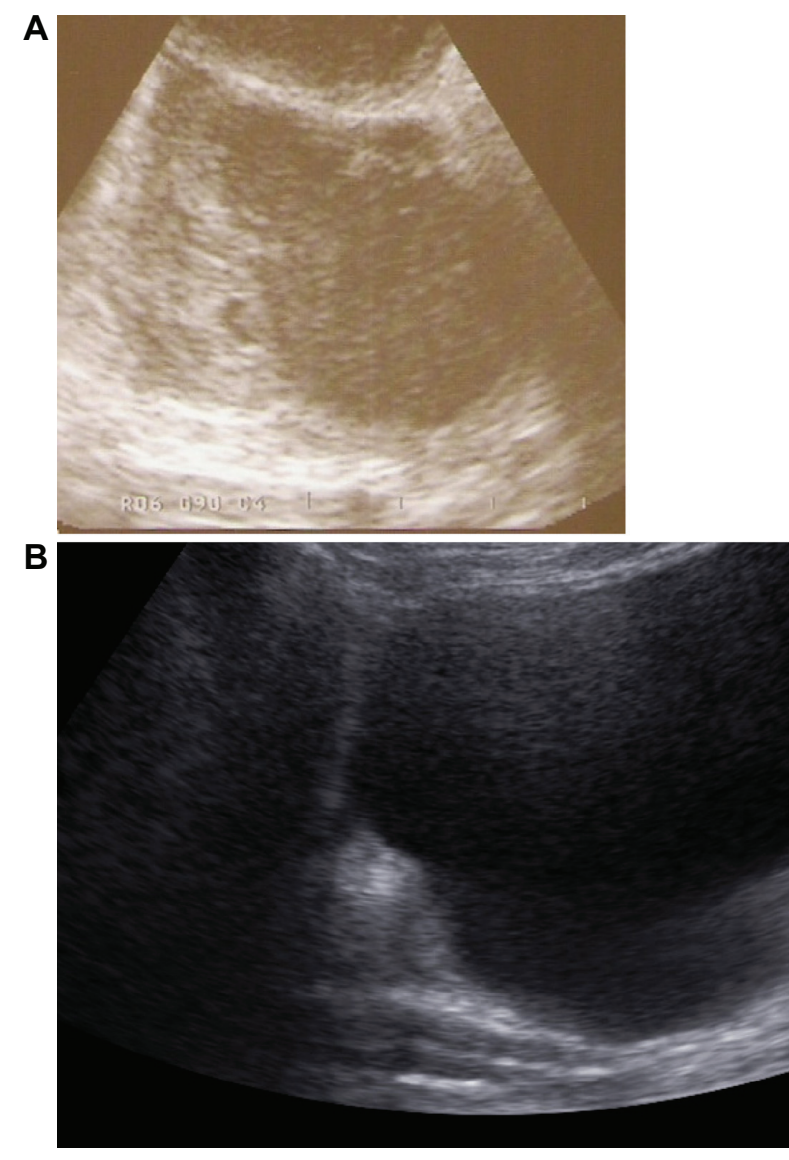

Figure 4 Endometriosis. A) Endometrioma. B) Deep infiltrated endometriosis (septum vesicovaginale).

Less specific adnexal masses and a positive human chorionic gonadotrophin pregnancy test together raise suspicion for an extrauterine or tubal pregnancy (Figure 5). The presence of a heart beating outside the uterus constitutes sonographic evidence of ectopic pregnancy. On the other hand, an empty uterine cavity detected by sonographic examination with a serum human chorionic gonadotrophin

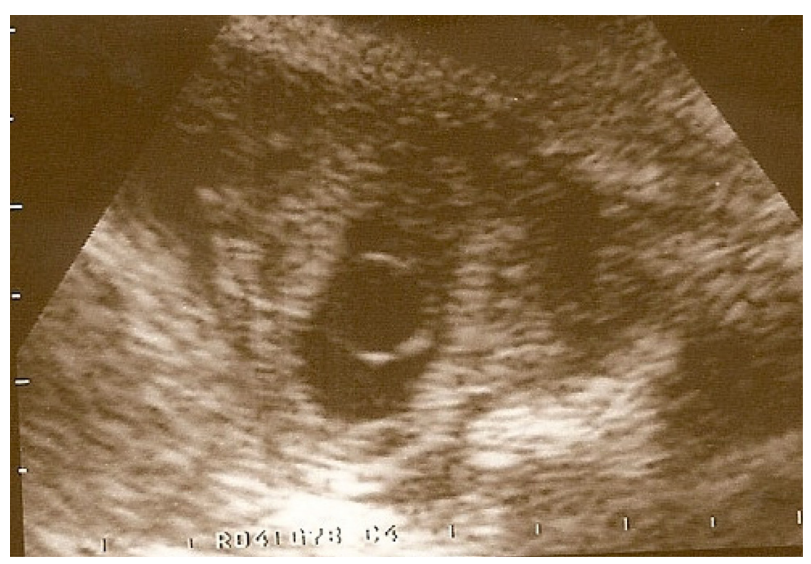

Figure 5 Tubal pregnancy. level above 1000 or $2000 \mathrm{IU} / \mathrm{L}$ allows us to establish the diagnosis of ectopic pregnancy with high probability. Often it is not possible to detect a small gestational sac in the very early stages of pregnancy. This condition is termed a pregnancy of unknown location. Where an ectopic pregnancy is developing, a blob sign, an inhomogenous mass near the ovary and bagel sign, an extrauterine gestational sac with a hyperechogenic halo, can be detected by sonography. We have previously reported our experience with pregnancy of unknown location. ${ }^{34}$

\section{Current examinations}

The new modalities of three-dimensional and color Doppler sonography have been used recently to enhance detection of malignant tissue. Re-evaluation of structural changes in adnexal masses and proposal of new scoring systems, as well as searching for additional imaging methods, become remarkable sonographic tools.

\section{Re-evaluation of adnexal masses}

Ultrasonographic characteristics of borderline ovarian tumors are different in mucinous and serous tumors. Echogenic fluid, multilocularity, and large diameter are more typical in borderline mucinous ovarian tumors, and papillary projections are associated with borderline serous ovarian tumors. ${ }^{35}$

\section{Novel three-dimensional/Doppler applications}

The use of color Doppler in the evaluation of flow location for the detection of adnexal malignancy is a reproducible method. ${ }^{36}$ Three-dimensional power Doppler VOCAL vascular indices (vascularization index and vascularization flow index in $1 \mathrm{cc}$ sphere) are recommended for predicting malignancy in vascular adnexal masses. ${ }^{37}$ Three-dimensional high-definition color Doppler flow imaging technique provides better resolution for depicting malignant ovarian tumor vessels (frequency of chaotic vessel arrangement) compared with color-pulsed Doppler and three-dimensional power Doppler sonography. ${ }^{38}$

\section{Scoring system innovations}

Specific sonographic structures and other parameters evaluated in a mathematic scoring system are used to distinguish between malignant and benign adnexal masses. Best known are the Sassone score, the Ovarian Tumor Index, and the Risk of Malignancy Index 3. A proposed new scoring system, ie, the Pelvic Masses Score, takes into account the ultrasound morphologic patterns, Doppler flussimetry of the pelvic mass, CA 125 serum level, and menopausal status of 
the patient. ${ }^{39}$ Another proposed new reporting system for classifying adnexal masses, ie, the Gynecologic Imaging Reporting and Data System, is based on sonographic findings. Pattern recognition analysis and color Doppler flow location determine the presumptive diagnosis. ${ }^{40}$

\section{Comparison with other imaging methods}

In the evaluation of adnexal masses, positron emission tomography/computed tomography does not provide additional information compared with expert transvaginal sonography. ${ }^{41}$ Combination of ultrasound with magnetic resonance imaging improves the accuracy in differentiation of benign from malignant ovarian lesions. ${ }^{42}$

The ISUOG congress in 2009 proposed four strategies to enhance the effect of sonography in the diagnosis of ovarian malignancy:

- Detection of typical adnexal structures, specific for each special type of ovarian malignancy performed by threedimensional sonography

- Evaluation of flow characteristics of malignant adnexal masses, ie, flow location, density and arrangement of new forming vessels performed by three-dimensional power Doppler

- Application of special scoring systems to detect malignancy

- Accumulation of diagnostic methods to enhance the probability of detection of ovarian malignancy.

\section{Endometriosis}

Recently, the availability of current sonographic methods in the diagnosis of endometriosis has been confirmed in comparison with other imaging methods. New sonographic methods, ie, mean gray value, are recommended. Three-dimensional imaging and Doppler seem to enhance the detection rate of endometriotic lesions. Visualization of malignant changes in endometrioma is a challenge.

\section{Confirmation of current methods}

Transvaginal sonography has high sensitivity for detection of severe pelvic endometriosis. ${ }^{43}$ Ultrasound examination is accurate in the detection of deep endometriotic nodes of the rectum and distal sigmoid wall. ${ }^{44}$ Sonovaginography is a well tolerated procedure, is cost-effective, and has higher accuracy in the detection of rectovaginal endometriosis. ${ }^{45}$ Transvaginal sonography can be useful in the diagnosis of vesicoperitoneal fistula due to deep infiltrating endometriosis as a cause of uroperitoneum. ${ }^{46}$ In the diagnosis of rectosigmoid endometriosis, magnetic resonance imaging shows similar accuracy in comparison with the ultrasonographic approach. ${ }^{47}$ Transvaginal sonography is an accurate imaging modality in detecting the presence of deeply infiltrating posterior endometriosis, with a better diagnostic performance than double-contrast barium enema. ${ }^{48}$

\section{Proposition of new methods}

Mean gray value of cyst content is higher in ovarian endometrioma than in other unilocular ovarian cysts. ${ }^{49}$

\section{Three-dimensional and Doppler exploitation}

In the near future, three-dimensional in deep infiltrating endometriosis could be an interesting field of research with a positive effect on everyday clinical practice. ${ }^{50}$

\section{Detection of malignant changes}

Malignant transformation of deep endometriosis should be considered in the differential diagnosis of pelvic lesions. ${ }^{51}$ Ovarian cancer arising in endometrioma is characterized by the presence of vascularized solid tissue detectable at ultrasound. This examination could be a valuable tool to recognize malignant transformation in endometrial cysts. ${ }^{52}$ The ISUOG congress in 2009 confirmed a current sonographic approach, especially the exclusive place of transvaginal sonography in the diagnosis of endometriosis and offers a vision of threedimensional and Doppler application not only for diagnosis of endometriosis, but for early detection of malignant transformation of this disease.

\section{Ectopic pregnancy}

Recently, new sonographic and biochemical methods for pregnancy of unknown location have been developed to improve the likelihood of correct diagnosis.

\section{Three-dimensional endometrial volume measurement}

The endometrial volume of the endometrium is higher in intrauterine pregnancy than in ectopic pregnancy. Threedimensional volumetric ultrasound assessment is of value in the prediction of both location and viability of pregnancies of unknown location. ${ }^{53}$

\section{Detection of intact endometrial midline echo}

Sonographic appearance of intact endometrial midline echo is a predictor of ectopic pregnancy in women with a pregnancy of unknown location. ${ }^{54}$ 


\section{Novel biochemical markers}

Levels of progesterone and inhibin A are lower and the level of IGFBP-1 is higher in pregnancies that spontaneously resolve. Therefore, these biochemical markers are useful in identifying which pregnancies of unknown location are going to resolve spontaneously. ${ }^{55}$ The ISUOG congress in 2009 showed that evaluation of structural characteristics and volumetric measurement of the endometrium as well as serum levels of different biomarkers can contribute to diagnosis of life-threatening ectopic pregnancy.

\section{Clinical research}

The main target of clinical sonographic research in the field of adnexal masses is to exclude a malignant process or to find a connection between malignancy and specific structure depiction, potentially with addition of serum oncomarkers. The "ovarian crescent sign" is of great interest in this regard. This sonographic structure, first described by Hillaby et al, ${ }^{56}$ is believed to be present in benign ovarian masses. It is defined as normal hypoechogenic ovarian tissue, with or without follicles, with a regular surface lying adjacent to an adnexal mass within the ovarian capsule. Yazbek et al confirmed the presence of the ovarian crescent sign in benign ovarian masses. ${ }^{57}$

Recent research by van Holsbeke et al has shown the presence of the ovarian crescent sign in $42 \%$ of benign ovarian masses, in $6 \%$ of invasive masses, and in $16 \%$ of borderline masses. This research confirms previous reports that the presence of the ovarian crescent sign decreases the likelihood of invasive malignancy in adnexal masses. However, it is a poor discriminator between benign and malignant adnexal masses. $^{58}$

The simple unilocular ovarian cyst, especially if $10 \mathrm{~cm}$ in diameter or less, carries a very low risk of malignancy. This is consistent with the study by Modesitt et al. ${ }^{59}$ On the contrary, complex or solid adnexal masses are associated with a significant risk of malignancy. This confirms the observation by $\operatorname{Im}$ et al. ${ }^{60}$

McDonald et al have shown enhanced sonographic diagnosis of adnexal masses in patients with complex or solid structures. An additional parameter for predicting risk of malignancy in patients with adnexal masses was measured, ie, the serum CA 125 level. In cases where the serum CA 125 level was $35 \mathrm{U} / \mathrm{mL}$, positive predictive values were $84.7 \%$ and $77.3 \%$ in patients with ovarian cancer Stages I and Stage II, respectively, and $98.6 \%$ in Stages III and IV. These results showed that patients with solid or complex ovarian tumors and an elevated serum CA 125 level are at high risk of ovarian malignancy. ${ }^{28}$

\section{Conclusion}

Gynecologic sonography is a viable, well developing entity. It is able to accept new challenges and incorporate them into the diagnostic process. New approaches in evaluation of sonographic structures can be seen in gynecologic pathology. Novel sonographic methods enable us to perform gynecologic diagnosis more exactly. Other imaging and biochemical methods can be helpful in this process. Clinical research is focused on sonographic methods to distinguish between benign and malignant gynecologic diseases.

\section{Disclosure}

The author reports no conflicts of interest in this work.

\section{References}

1. van den Bosch $\mathrm{T}$, Luts $\mathrm{J}$, Bourne $\mathrm{T}$, et al. Interobserver agreement on reporting uterine intracavity lesions at gel infusion sonography. Ultrasound Obstet Gynecol. 2009;34 Suppl 1:158.

2. Leone FPG, Timmerman D, Bourne T, et al. Terms, definitions and measurements to describe the sonographic features of the endometrium and intrauterine lesions: A consensus opinion from the International Endometrial Tumour Analysis (IETA) group. Ultrasound Obstet Gynecol. 2010;35:103-112.

3. Bermejo C, Martinez Ten P, Cantarero C, et al. Three dimensional ultrasound in the diagnosis of Mullerian duct anomalies and concordance with magnetic resonance imaging. Ultrasound Obstet Gynecol. 35;2010:593-601.

4. van den Bosch T, Luts J, Bourne T, et al. Comparison of gel instillation sonography (GIS) with unenhanced ultrasound in the diagnosis of uterine intracavity lesion. Ultrasound Obstet Gynecol. 2009;34 Suppl 1:19.

5. Werbrouck E, van den Bosch T, Veldman J, et al. Saline infusion versus gel instillation sonography: A prospective cohort study. Ultrasound Obstet Gynecol. 2009;34 Suppl 1:19-20.

6. Emanuel MH, Tromp I, Betlem M. The diagnostic accuracy of gel instillation sonography (GIS) compared with saline infusion sonography (SIS): A randomised controlled trial. Ultrasound Obstet Gynecol. 2009;34 Suppl 1:20.

7. Bij de Vaate M, Brolmann H, van der Slikke JW, et al. Gel instillation sonography (GIS) and saline infusion sonohysterography (SIS): Comparison of diagnostic accuracy. Ultrasound Obstet Gynecol. 2009; 34 Suppl 1:161.

8. Negm S, Kamel R, Momtaz M, et al. Classification of submucous myomas using 3D multislice saline infusion sonohysterography and its correlation with hysteroscopy. Ultrasound Obstet Gynecol. 2009; 34 Suppl 1:158.

9. Mavrelos D, Ben Nagi J, Lee C, et al. Three dimensional saline infusion sonohysterography for the preoperative prediction of submucous fibroid respectability. Ultrasound Obstet Gynecol. 2009;34 Suppl 1: $159-160$.

10. van den Bosch T, Luts J, Bourne T, et al. The influence of gel-infusion on the vascularity of endometrial polyps. Ultrasound Obstet Gynecol. 2009;34 Suppl 1:160.

11. Exacoustos C, Vaquero E, Romeo V, et al. Three dimensional sonographic assessment of septate uterus: Correlation between morphology, volume and vascularity of the septum and reproductive outcome. Ultrasound Obstet Gynecol. 2009;34 Supp1 1:29. 
12. Jalinik K, Chmielevski G, Romejko-Wolniewicz E, et al. The diagnostic value of transvaginal sonography compared to hysteroscopy in endometrial polyp and submucous fibroid detection. Ultrasound Obstet Gynecol. 2009;34 Suppl 1:161.

13. Momtaz M, Ebrashy A, Aboulghar M, et al. 3D sonohysterography versus $3 \mathrm{D}$ ultrasound examination of the coronal plane of the uterus in fertile women with suspected uterine cavity lesion or anomalies. Ultrasound Obstet Gynecol. 2009;34 Suppl 1:28.

14. Morel O, Malartic C, Rivain A, et al. Interest of pelvic magnetic resonance imaging for the pretherapeutic evaluation of symptomatic uterine fibroids: Evaluation in 68 cases. Ultrasound Obstet Gynecol. 2009; 34 Suppl 1:159.

15. Exacoustos C, Di-Giovanni A, Szabolcz B, et al. Feasibility of automated sonographic tubal patency evaluation using threedimensional Coded Contrast Imaging. Ultrasound Obstet Gynecol. 2009;34 Suppl 1:101.

16. Alcázar JL, Castillo G, Míngues JÁ, et al. Endometrial blood flow mapping using transvaginal power Doppler sonography in women with postmenopausal bleeding and thickened endometrium. Ultrasound Obstet Gynecol. 2003;21:583-588.

17. Timmerman D, Verguts J, Konstantonovic ML, et al. The pedicle artery sign based on sonography with color Doppler imaging can replace second-stage test in women with abnormal bleeding. Ultrasound Obstet Gynecol. 2003;22:166-171.

18. Cil AP, Tulunay G, Kose MF, et al. Power Doppler properties of endometrial polyps and submucosal fibroids: A preliminary observation study in women with known intracavitary lesions. Ultrasound Obstet Gynecol. 2010;35:233-237.

19. Opolskiene G, Sladkevicius P, Jakubkiene L, et al. Three dimensional ultrasound imaging for discrimination between benign and malignant endometrium in women with postmenopausal bleeding and sonographic endometrial thickness of at least $4.5 \mathrm{~mm}$. Ultrasound Obstet Gynecol. 2010;35:94-102.

20. Veldman J, van den Bosch T, Werbrouck E, et al. Endometrial thickness measurement with and without fluid contrast ultrasound. Ultrasound Obstet Gynecol. 2009;34 Suppl 1:21.

21. Leone F, Marciante C, Crepaldi A, et al. Saline contrast sonohysterography with liquid-based endometrial cytology in post-menopausal women. Ultrasound Obstet Gynecol. 2009;34 Suppl 1:53-54.

22. Alberto R, Calcagno A, Vascotto L, et al. The diagnostic value of endometrial volume and vascularity measurement by transvaginal three-dimensional ultrasonography and power Doppler angiography in patients with postmenopausal bleeding. Ultrasound Obstet Gynecol. 2009;34 Suppl 1:158.

23. Alcazar J, Albela S, Galvan R. Assessing myometrial infiltration by endometrial cancer: Uterine virtual navigation with three-dimensional ultrasound. Ultrasound Obstet Gynecol. 2009;34 Supp1 1:53.

24. van den Bosch T, Domali E, Werbrouck E, et al. VOCAL in the evaluation of the endometrium: A pilot study. Ultrasound Obstet Gynecol. 2009;34 Suppl 1:160.

25. Albela S, Martinez-Roman S, Pahisa J, et al. Myometrial invasion of endometrial cancer: Comparison of 3D US and RM imaging. Ultrasound Obstet Gynecol. 2009;34 Suppl 1:53.

26. Opolskiene G, Sladkievicius P, Valentin L. Ultrasound assessment of endometrial morphology and vascularity to predict endometrial malignancy in women with postmenopausal bleeding and sonographic endometrial thickness $\geq 4.5 \mathrm{~mm}$. Ultrasound Obstet Gynecol. 2007; 30:332-340.

27. Galván R, Mercé L, Jurado M, et al. Three-dimensional power Doppler angiography in endometrial cancer: Correlation with tumour characteristics. Ultrasound Obstet Gynecol. 2010;35:723-729.

28. McDonald J, Doran S, DeSimone $\mathrm{CH}$, et al. Predicting risk of malignancy in adnexal masses. Obstet Gynecol. 2010;115:687-694.

29. Ameye L, van Holsbeke C, Testa A. Intercenter variability in ultrasound features of malignant and benign adnexal masses. Ultrasound Obstet Gynecol. 2009;34 Suppl 1:48-49.
30. Kudla MJ, Alcazar J, Timor-Tritsch I, et al. Comparison of volume evaluation of pathological and normal ovaries obtained with the use of VOCAL 3D software versus the " 3 distances" method. Ultrasound Obstet Gynecol. 2009;34 Supp1 1:49.

31. Valentin LI, Ameye L, Savelli L. Unilocular solid adnexal masses with papillary projections but not other solid components: Is there a logistic regression model that can help? Ultrasound Obstet Gynecol. 2009; 34 Suppl 1:48.

32. van Holsbeke C, van Calster B, Guerriero S, et al. Endometriomas: Their ultrasound characteristics. Ultrasound Obstet Gynecol. 2010; 35:730-740

33. Gardanis K, Zdichavsky M, Kramer M, et al. Minimally invasive surgery for deep infiltrating endometriosis in the rectovaginal septum. Geburtsh Frauenheilk. 2010;70:194-200.

34. Drobný J. Sonography in the management of symptomatic pregnancies of unknown location. Bratisl Med J. 2008;109:254-259.

35. Goldberg Y, Lavie O, Keidar R, et al. Ultrasonographic characteristics of borderline ovarian tumours - 5-year experience of a single institution. Ultrasound Obstet Gynecol. 2009;34 Suppl 1:140-141.

36. Guerriero S, Alcazar J, Pascual MA, et al. Intraobserver and interobserver agreement of color Doppler in adnexal malignancy. Ultrasound Obstet Gynecol. 2009;34 Suppl 1:141.

37. Alcazar J, Kudla MJ. Does spherical sample size affect the performance of 3D power Doppler indexes for predicting malignancy in vascularized adnexal masses? Ultrasound Obstet Gynecol. 2009;34 Suppl 1:44.

38. Czekierdowski A, Smolen A, Kotarski J. Three-dimensional highdefinition color Doppler flow imaging and "vascular" tumour biopsy to assess complex ovarian masses: A preliminary experience. Ultrasound Obstet Gynecol. 2009;34 Suppl 1:50.

39. Alberto R, Capodicasa V, Fachechi G, et al. The Pelvic Masses Score (PMS): A proposed new scoring system to evaluate pelvic masses. Ultrasound Obstet Gynecol. 2009;34 Suppl 1:139.

40. Amor F, Vaccaro H, León M, et al. GI-RADS: A new proposal for classifying adnexal masses based on sonographic findings. Ultrasound Obstet Gynecol. 2009;34 Suppl 1:141-142.

41. Giunchi S, Kuleva M, Ghi T, et al. Comparison of the diagnostic accuracy of expert transvaginal ultrasound and PET/CT in the evaluation of adnexal masses. Ultrasound Obstet Gynecol. 2009;34 Suppl 1:43-44.

42. Pinschke M, Gnann D, Smekal I, et al. Asymptomatic adnexal masses: Accuracy of characterization with ultrasound scoring systems, color Doppler sonography, 3D-ultrasound, and MR imaging. Ultrasound Obstet Gynecol. 2009;34 Suppl 1:139.

43. Holland TK, Yazbek J, Hoo W, et al. The value of transvaginal ultrasound in assessing of severity of pelvic endometriosis. Ultrasound Obstet Gynecol. 2009;34 Suppl 1:23.

44. van Schoubroeck D, Meuleman C, van den Bosch T, et al. Ultrasound in the detection of rectal- and distal sigmoid involvement of deep endometriosis. Ultrasound Obstet Gynecol. 2009;34 Suppl 1:24.

45. Cosmi E, Dessole S, Saccardi C, et al. Deep infiltrating endometriosis: Sonovaginography or nuclear magnetic resonance? Ultrasound Obstet Gynecol. 2009;34 Suppl 1:24.

46. Guerriero S, Ajossa S, Pilloni M, et al. Transvaginal sonography in the diagnosis of vesico-peritoneal fistula due to deep infiltrating endometriosis as a cause of uroperitoneum. Ultrasound Obstet Gynecol. 2009; 34 Suppl 1:284.

47. Guerriero S, Saba I, Ajossa S, et al. Magnetic resonance imaging and transvaginal modified ultrasonography in the diagnosis of rectosigmoid endometriosis. Ultrasound Obstet Gynecol. 2009;34 Suppl 1:24.

48. Savelli L, Manuzzi L, Pollastri P, et al. Transvaginal sonography and double-contrast barium enema in the diagnosis of intestinal deeply infiltrating endometriosis. Ultrasound Obstet Gynecol. 2009; 34 Suppl 1:25.

49. Alcazar J, León M, Guerriero S. Assessment of cyst content by using mean gray value for discriminating ovarian endometrioma from other unilocular cyst in premenopausal women. Ultrasound Obstet Gynecol. 2009;34 Suppl 1:25. 
50. Guerriero S, Alcazar J, Ajossa S, et al. The potential of threedimensional (3D) ultrasound for the detection of deep endometriosis. Ultrasound Obstet Gynecol. 2009;34 Suppl 1:281.

51. Guerriero S, Piras B, Ajossa S, et al. Extrauterine undifferentiated endometroid stromal sarcoma arising in endometriosis of the rectovaginal septum of a menopausal patient with recent onset of pelvic pain. Ultrasound Obstet Gynecol. 2009;34 Suppl 1:160.

52. Testa A, van Holsbeke C, Licameli A, et al. Ovarian cancer arising in endometrioma: Ultrasound findings. Ultrasound Obstet Gynecol. 2009;34 Suppl 1:50.

53. Bignardi T, Alhamdan D, Riemke J, et al. Endometrial volume measurements by transvaginal three-dimensional ultrasound for the prediction of outcome in the pregnancies of unknown location. Ultrasound Obstet Gynecol. 2009;34 Suppl 1:16.

54. Hoo W, Jones B, Backos M, et al. Does an intact endometrial midline echo predict ectopic pregnancy in women with pregnancies of unknown location. Ultrasound Obstet Gynecol. 2009;34 Suppl 1:16.

55. Chetty M, Sawyer E, Dew T, et al. Novel biochemical markers to predict spontaneous resolution of pregnancies of unknown location. Ultrasound Obstet Gynecol. 2009;34 Suppl 1:16.
56. Hillaby K, Aslam N, Salim R, et al. The value of detection of normal ovarian tissue (the "ovarian crescent sign") in the differential diagnosis of adnexal masses. Ultrasound Obstet Gynecol. 2004;23:63-67.

57. Yazbek J, Aslam N, Tailor A, et al. A comparative study of the risk of malignancy index and the "crescent ovarian sign" for the diagnosis of invasive ovarian cancer. Ultrasound Obstet Gynecol. 2006; 28:320-324.

58. van Holsbeke C, van Belle V, Leone FPG, et al. Prospective external validation of the ovarian crescent sign as a single ultrasound parameter to distinguish between benign and malignant adnexal pathology. Ultrasound Obstet Gynecol. 2010;36:81-87.

59. Modesitt SC, Pavlik EJ, Ueland FR, et al. Risk of malignancy in unilocular ovarian cyst tumours less than $10 \mathrm{~cm}$ in diameter: A long-term follow-up study. Obstet Gynecol. 2003;102:594-599.

60. Im S, Gordon A, Buttin B, et al. Validation of referral guidelines for women with pelvic masses. Obstet Gynecol. 2005;105:34-41.

Reports in Medical Imaging

\section{Publish your work in this journal}

Reports in Medical Imaging is an international, peer-reviewed, open access journal publishing original research, reports, reviews and commentaries on all areas of medical imaging. The manuscript management system is completely online and includes a very quick and fair peer-review system, which is all easy to use.

\section{Dovepress}

Visit http://www.dovepress.com/testimonials.php to read real quotes from published authors. 